\title{
¿Hemoperitoneo en diálisis peritoneal, un signo de alarma?
}

\author{
Sylvia Quiñones Sussman ${ }^{1}$, Carolina Larrarte Arenas ${ }^{1}$, Freddy Ardila Celis ${ }^{2}$ \\ 1 Nefróloga, RTS-Agencia Santa Clara, unidad RTS-Agencia Santa Clara. Bogotá, Colombia..

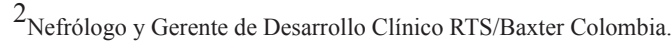

\section{Resumen}

El hemoperitoneo es una complicación de la diálisis peritoneal. Su diagnóstico diferencial es amplio y el enfoque se basa en el cuadro clínico y su severidad. Es necesario evaluar todas las causas del hemoperitoneo y tener en cuenta que tienen manifestaciones diferentes y que algunas arriesgan la vida del paciente. A continuación se describe un caso de un paciente con largo tiempo en diálisis peritoneal con hemoperitoneo, en quien el estudio sugiere peritonitis calcificante como enfermedad de base.

Palabras clave: hemoperitoneo, diálisis peritoneal, peritonitis calcificante, peritonitis esclerosante encapsulada.

\section{¿Hemoperitoneum in peritoneal dialysis, a red flag?}

\section{Summary}

Hemoperitoneum is a complication of peritoneal dialysis. The differential diagnosis is wide and the approach is based on clinical presentation and severity. It is important to evaluate all the causes of hemoperitoneum and to consider that it may be life risking. This is a case of a patient on long term peritoneal dialysis with hemoperitoneum, whose study showed calcifying peritonitis as the underlying condition.

Key words: hemoperitoneum, peritoneal dialysis, calcifying peritonitis, sclerosing encapsulating peritonitis.

\section{Introducción}

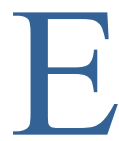
hemoperitoneo es una complicación común enlos pacientes en diálisis peritoneal, es más frecuente en mujeres y atribuible a causas ginecológicas, pero puede presentarse como consecuencia de múltiples etiologías, una de ellas, es la peritonitis calcificante, en la cual el hemoperitoneo es causado por retracciones de los vasos o adhesiones entre órganos intraabdominales. La peritonitis calcificante puede asociarse a peritonitis esclerosante encapsulada que, si bien es infrecuente, genera gran morbimortalidad en el paciente en diálisis peritoneal ${ }^{1,2}$. 


\section{Descripción de caso}

Paciente masculino de 55 años, con enfermedad poliquística renal, en diálisis peritoneal durante 18 años; con antecedentes de enfermedad coronaria e hiperparatiroidismo.

Durante los últimos 6 años en diálisis peritoneal se mantuvo como transportador rápido para creatinina y promedio para glucosa, al momento de la presentación del cuadro que se describe, presentaba ultrafiltración promedio mayor a 1 litro por día, sin función renal residual y sin signos de sobrecarga hídrica. No se encontraron antecedentes de síntomas gastrointestinales, pérdida de peso ni infecciones, no refería antecedente de peritonitis asociada a diálisis peritoneal. En la tabla 1 se describen los resultados de los exámenes de laboratorio tomados en su último control en la unidad renal ( 25 días antes de la consulta).

El paciente consulta por presentar líquido peritoneal de aspecto hemático en los últimos 2 días, sin dolor abdominal. Tres horas antes del ingreso a urgencias, presenta síncope. Años atrás había presentado 2 episodios autolimitados de hemoperitoneo. Al examen físico presentaba presión arterial de 90/50 $\mathrm{mmHg}$, frecuencia cardiaca de 105 latidos por minuto y 18 respiraciones por minuto. A la inspección del abdomen, el catéter peritoneal y su orificio se encontra-

\begin{tabular}{|ll|}
\hline \multicolumn{2}{|c|}{ Tabla 1. } \\
\hline \multicolumn{2}{|c|}{ Laboratorios del paciente. } \\
\hline Laboratorio & Resultado \\
\hline Hemoglobina & $10 \mathrm{gr} / \mathrm{dL}$ \\
Hematocrito & $29,9 \%$ \\
Ferritina & $121,3 \mathrm{pg} / \mathrm{mL}$ \\
TSAT & $10 \%$ \\
Calcio & $9,04 \mathrm{mg} / \mathrm{dL}$ \\
Fósforo & $5,4 \mathrm{mg} / \mathrm{dL}$ \\
PTH & $1655 \mathrm{pg} / \mathrm{mL}$ \\
Albúmina & $2,6 \mathrm{gr} / \mathrm{dL}$ \\
\hline TSAT (por sus siglas en inglés): porcentaje de saturación de la \\
transferrina. \\
PTH (por sus siglas en inglés): hormona paratiroidea. \\
PCR: proteína C reactiva.
\end{tabular}

ban en buen estado, no tenía dolor a la palpación ni signos de irritación peritoneal. No tenía otros hallazgos relevantes al examen físico. Fue hospitalizado con diagnóstico de hemoperitoneo. Los exámenes diagnósticos mostraron una hemoglobina de $6.2 \mathrm{gr} /$ $\mathrm{dL}$, por lo que requirió transfusión de glóbulos rojos, logrando estabilidad hemodinámica. Dada la presencia de anemia y el compromiso hemodinámico, se consideró descartar lesión abdominal vascular o de órgano intraabdominal, por lo que se realizó angiotomografía de abdomen, cuyas imágenes documentaron extensas calcificaciones mesentéricas y peritoneales y múltiples quistes renales con algunas calcificaciones, sin signos de ruptura ni sangrado (figura 1).

El paciente persistía con anemia y líquido peritoneal hemático por lo que se realizó gammagrafía con eritrocitos marcados, la cual fue negativa. Una vez estabilizada la hemoglobina y con mejoría de las características del líquido peritoneal, el paciente es dado de alta. Los signos vitales de egreso eran presión arterial de 140/90 mmHg, frecuencia cardiaca de 84 latidos por minuto y frecuencia respiratoria de 18 respiraciones por minuto. Al día siguiente del egreso hospitalario consulta a la unidad renal por hemoperitoneo y lo encuentran con presión arterial de 80/40 mmHg y frecuencia cardiaca de 112 latidos por minuto, nuevamente se hospitaliza y se realiza laparoscopia abdominal que evidencia gran hematoma contenido (2 litros) sobre el epiplón mayor del ángulo esplénico del colon, ligamento gastroesplénico y espacio subfrénico izquierdo, adicionalmente, se evidencia sangrado activo de la rama arterial frénica inferior izquierda cerca del hiato esofágico y engrosamiento severo de las asas intestinales delgadas por peritoneo fibrosado; se liga el vaso y se retira el catéter de diálisis peritoneal.

El cuadro clínico se resuelve y el paciente se traslada a terapia de hemodiálisis y se da de alta hospitalaria. La evolución del paciente, 11 meses después de este cuadro, es adecuada, continúa en hemodiálisis, no ha vuelto a presentar síntomas gastrointestinales.

\section{Revisión de la literatura}

El hemoperitoneo es un síntoma que pueden presen- 


\section{Figura 1.}

Corresponde a las imágenes de angiotomografía de abdomen en corte coronal y axial en donde se evidencia importante calcificación alrededor de las asas intestinales.
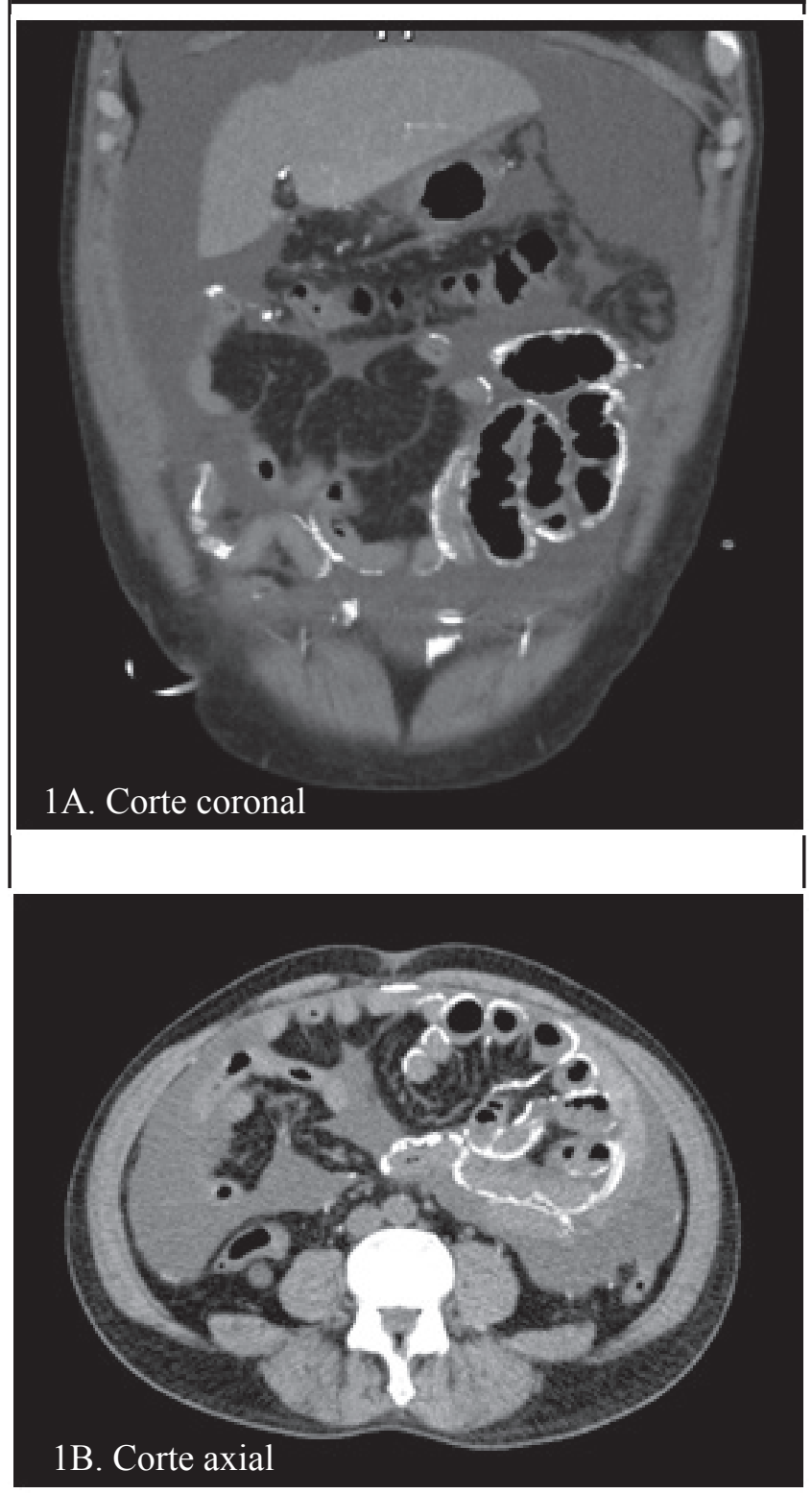

tar los pacientes en diálisis peritoneal, se requieren tan sólo $2 \mathrm{~mL}$ de sangre en una bolsa de drenaje de líquido peritoneal de 1 litro para que cambie la coloración de este ${ }^{1}$. Existen múltiples causas de hemoperitoneo y dentro de las más frecuentes se encuentran las asociadas a la inserción del catéter de diálisis peritoneal y la menstruación en las mujeres. En el enfoque de un paciente con hemoperitoneo se debe tener en cuenta si es autolimitado o persistente y la severidad del cuadro clínico. Es necesario descartar las diferentes etiologías de hemoperitoneo como son las relacionadas con el catéter u otros procedimientos, procesos infecciosos, lesiones vasculares o de órganos intraabdominales, causas obstétricas o ginecológicas, entre otros ${ }^{1,3-5}$.

La peritonitis calcificante es otra causa menos frecuente de hemoperitoneo. Esta entidad fue descrita por Marichalet al. ${ }^{6}$, quienes reportan 2 pacientes en diálisis peritoneal con dolor abdominal, síntomas de obstrucción intestinal incompleta y calcificaciones en el peritoneo. Histológicamente, el peritoneo parietal de estos pacientes mostraba engrosamiento fibroso, bandas de osificación, depósitos de calcio y pocas células. Estos pacientes se dializaban con soluciones con acetato por lo que se consideró un agente etiológico de la calcificación del peritoneo. Uno de los pacientes tenía hiperparatiroidismo y había presentado hemoperitoneo. Los pacientes evolucionaron favorablemente después del cambio de terapia a hemodiálisis. Estos 2 casos y otro reportado en la literatura, sugerían que esta patología era de buen pronóstico ${ }^{6,7}$.

La causa de la peritonitis calcificante es incierta, pero se han postulado diferentes etiologías como son los componentes de la solución de diálisis peritoneal como el acetato, episodios repetidos de peritonitis bacteriana, el mismo hemoperitoneo que puede acelerar la calcificación y las alteraciones minerales óseas de los pacientes en diálisis por alteración del eje fosfato-calcio-hormona paratiroidea ${ }^{6,8,9}$. Se ha postulado que el hemoperitoneo asociado a peritonitis calcificante puede ser causado por laceraciones de las adhesiones en el compartimento intraabdominal (por ejemplo, adhesiones del peritoneo a los órganos o de los órganos al diafragma), por retracción de los vasos por placas calcificadas circundantes o por la misma calcificación vascular que debilita la pared del vaso ${ }^{10}$. Dentro de las causas arteriales de hemoperitoneo se encuentran también la lesión de los vasos por el catéter de diálisis peritoneal o la ruptura espontánea de vasos anormales con aneurismas o pseudoaneurismas ${ }^{11}$. Los vasos implicados usualmente son la arteria esplénica y las renales ${ }^{11}$; 
sin embargo, se encuentran casos reportados de la arteria gastroepiploica $^{12}$, hepática ${ }^{13}$, gástrica ${ }^{14}$ y mesentérica superior ${ }^{11}$.

La peritonitis calcificante se asocia, ocasionalmente, a peritonitis esclerosante encapsulada $(\mathrm{PEE})^{10}$. Esta asociación, reportada hace varios años en la literatura, hace dudar acerca del buen pronóstico de la primera entidad. La PEE es una de las complicaciones más serias de la diálisis peritoneal y se caracteriza por síntomas de obstrucción intestinal, fibrosis del peritoneo y alta morbimortalidad. Las asas intestinales se encapsulan y adhieren dentro del peritoneo esclerosado. El diagnóstico de la PEE requiere la presencia de síntomas de obstrucción intestinal con o sin inflamación sistémica y calcificaciones o intestino encapsulado. De acuerdo con los hallazgos clínicos y patológicos, la PEE se divide en 4 períodos (tabla 2$)^{2}$.

El diagnóstico e intervención temprana de la PEE impacta el pronóstico, por lo que cobra importancia el reconocimiento de los pacientes en fase pre-PEE; sin embargo, existe dificultad para ello dado que no hay una definición universalmente aceptada de la fase pre-PEE, los signos de la fase pre-PEE no son patognomónicos de esta y no todos los pacientes en esta fase progresan al cuadro clínico de $\mathrm{PEE}^{15}$. Adicionalmente, no hay disponibilidad de marcadores clínicos ni de imaginología que predigan la progresión a PEE de los pacientes en diálisis peritoneal ${ }^{16}$.

Las conductas terapéuticas de la peritonitis calcificante no están claramente establecidas. Teniendo en cuenta las posibles etiologías, habría que minimizar la frecuencia de peritonitis, usar soluciones con baja concentración de glucosa y realizar intervención de la enfermedad mineral ósea ${ }^{5,15}$. Algunos sugieren cambio de terapia dialítica a hemodiálisis ${ }^{5}$. A pesar de que los pacientes de los reportes originales presentaron mejoría de los síntomas al suspender la diálisis peritoneal, hay datos de pacientes que progresan a PEE y presentan cuadros de obstrucción intestinal posterior al cambio a hemodiálisis ${ }^{10}$. Esto ha sido explicado por la remoción de fibrina por la diálisis y la ausencia del líquido peritoneal que favorece el contacto entre las asas intestinales y, por lo tanto, su adhesión ${ }^{15}$.

A mayor tiempo en diálisis peritoneal, aumenta el riesgo de PEE; sin embargo, hay pacientes con largo tiempo en diálisis peritoneal con estabilidad de la función de la membrana y sin desarrollar este cuadro $^{2}$. No está establecido el momento preciso en el que deba suspenderse la diálisis peritoneal. Esta decisión debe ser individualizada y debe tenerse en cuenta el cambio en el transporte peritoneal, la falla de ultrafiltración y la necesidad de soluciones hipertónicas para controlar el volumen, el estado nutricional del paciente, los signos de inflamación y cuadros de peritonitis ${ }^{15}$.

En el año 2008, fueron publicados 3 casos clínicos en la revista Therapeutic Apheresis and Dialysis ${ }^{10}$, en los cuales se documentó calcificación peritoneal en pacientes que se encontraban en diálisis peritoneal por largo tiempo (más de 7 años). En el primer caso, el paciente presentaba hiperparatiroidismo secundario, requería soluciones con alta concentración de glucosa para lograr ultrafiltración y refería sín-

\begin{tabular}{|ll|}
\hline \multicolumn{1}{|c|}{ Tabla 2. } \\
\hline \multicolumn{1}{|c|}{ Estadios de la peritonitis esclerosante encapsulada (PEE) } \\
\hline Estadios & Hallazgos \\
\hline 1. Asintomático (período pre-PEE) & $\begin{array}{l}\text { Falla de ultrafiltración, transporte rápido, hipoproteinemia, hemoperitoneo, ascitis, } \\
\text { calcificaciones peritoneales. }\end{array}$ \\
2. Período inflamatorio & $\begin{array}{l}\text { Hemoperitoneo, fiebre, ascitis, pérdida de peso, hiporexia, diarrea, elevación de proteína C } \\
\text { reactiva. }\end{array}$ \\
3. Período progresivo o encapsulante & $\begin{array}{l}\text { Signos y síntomas de íleo (náuseas, vómito, dolor abdominal, constipación, masa } \\
\text { abdominal, ascitis) }\end{array}$ \\
4. Período obstructivo & Anorexia, obstrucción intestinal completa, masa abdominal
\end{tabular}


tomas abdominales y cuadros de hemoperitoneo intermitente. Las imágenes demostraron calcificación del peritoneo parietal y de la superficie hepática sin obstrucción intestinal. El paciente requirió traslado a hemodiálisis y a los 5 años de estar en esta terapia no había presentado síntomas de obstrucción intestinal ni cambios en las imágenes en cuanto a la calcificación. Los otros 2 casos descritos, correspondían a pacientes que también tenían hiperparatiroidismo secundario, antecedente de peritonitis y habían requerido cambio de diálisis peritoneal a hemodiálisis por falla de la membrana para lograr ultrafiltración $\mathrm{y}$, posterior a este cambio, presentaron síntomas gastrointestinales compatibles con obstrucción intestinal. En las imágenes se detectó calcificación del intestino y del peritoneo parietal, sugiriendo diagnóstico de PEE.

Los autores de este artículo resaltan que aunque la calcificación peritoneal se considera un signo temprano de PEE, esta no siempre se presenta concomitantemente. Sin embargo, concluyen que debe realizarse monitorización de la calcificación peritoneal incluso después de suspender la diálisis peritoneal y que es necesario estar atentos ante la presencia de otros signos por la posibilidad de progresar a $\mathrm{PEE}^{10}$.

\section{Discusión}

El caso que se presentoì anteriormente corresponde a un paciente con 18 anPos en diailisis peritoneal, con hemoperitoneo persistente y severo. Por el compromiso hemodinaìmico, el descenso significativo de la hemoglobina y los antecedentes del paciente, se debiìa considerar la ruptura de un quiste renal como posible causa del hemoperitoneo; sin embargo, este era de origen vascular debido a sangrado de la ar- teria freìnica inferior. Lo relevante del caso clínico corresponde a los hallazgos de calcificaciones extensas en mesenterio y peritoneo, que sumado al largo tiempo en diálisis peritoneal, el hemoperitoneo y el hiperparatiroidismo, podría corresponder a un cuadro de peritonitis calcificante.

Al analizar el caso descrito y teniendo en cuenta la asociación entre peritonitis calcificante y PEE, el paciente podría encontrarse en la fase pre-PEE, en la que aún las manifestaciones clínicas del cuadro no se han presentado. El paciente no presentaba síntomas de obstrucción intestinal ni falla de ultrafiltración; sin embargo, cursaba con hemoperitoneo, calcificaciones peritoneales y transporte peritoneal rápido. El transporte peritoneal rápido puede deberse al largo tiempo en diálisis peritoneal y los cambios histológicos del peritoneo expuesto a soluciones de diálisis durante un período significativo; sin embargo, hay que tener en cuenta que el cambio de transporte peritoneal a rápido puede ser un marcador temprano de PEE.

En este caso, se realizó cambio de modalidad a hemodiálisis. En el momento, el paciente lleva 11 meses en esta terapia, sin presentar síntomas gastrointestinales, marcadores de inflamación ni desnutrición. El hemoperitoneo fue el síntoma principal de nuestro paciente, que puede ser considerado un signo de alarma de peritonitis calcificante, ya que su estudio nos permitió documentar los hallazgos concernientes a esta patología. Teniendo en cuenta la discusión anotada previamente, es importante estar vigilantes ante los síntomas de progresión a PEE en el paciente y realizar intervención oportuna para impactar en el pronóstico.

\section{Conclusión}

El hemoperitoneo es una complicación de los pacientes en diálisis peritoneal. Existen causas de hemoperitoneo que amenazan la vida y que requieren intervención inmediata como las lesiones vasculares, de órganos intraabdominales, entre otros. Por esto, es importante realizar el abordaje diagnóstico basado en la persistencia y severidad de este.

Sin embargo, hay que tener en cuenta que el hemoperitoneo puede ser un síntoma de una condición subyacente, como la peritonitis calcificante, que aunque su presentación es larvada, en caso de no detectarse ni intervenirse, puede contribuir a la presentación de $\mathrm{PEE}$, que empeora el pronóstico de los pacientes en diálisis peritoneal, en cuanto a calidad de vida, morbilidad y mortalidad. En pacientes en diálisis peritoneal por períodos prolongados, hay que estar vigilantes ante la presencia de hemoperitoneo y cambio en el comportamiento de la membrana peritoneal, dado que podrían ser considerados marcadores tempranos de PEE. 


\section{Conflicto de intereses y financiación}

Los autores declaran que no existen conflictos de interés. No existen fuentes de financiación en la publicación de este reporte de caso.

\section{Bibliografía}

1. Lew SQ. Hemoperitoneum: bloody peritoneal dialysate in ESRD patients receiving peritoneal dialysis. Perit Dial Int. 2007;27(3):226-33.

2. Nakamoto H. Encapsulating peritoneal sclerosis-a clinician's approach to diagnosis and medical treatment. Perit Dial Int. 2005;25Suppl 4:S30-8.

3. Lew SQ. Hemoperitoneum: bloody peritoneal dialysate in ESRD patients receiving peritoneal dialysis. Perit Dial Int. 2007;27(3):226-33.

4. Tse KC, Yip PS, Lam MF, Li FK, Choy BY, Chan TM, et al. Recurrent hemoperitoneum complicating continuous ambulatory peritoneal dialysis. Perit Dial Int. 2002;22(4):488-91.

5. Bargman JM. Non infectious complications of peritoneal dialysis. In: R. Khanna, R.T. Krediet Editors. Nolph and Gokal's Textbook of Peritoneal Dialysis. New York: Springer; 2009. p. 571-609.

6. Marichal JF, Faller B, Brignon P, Wagner D, Straub P. Progressive calcifying peritonitis: a new complication of CAPD? Report of two cases. Nephron. 1987;45:229-32.

7. Francis DM, Busmanis I, Becker G. Peritoneal calcification in a peritoneal dialysis patient: a case report. Perit Dial Int. 1990;10:237-40.

8. Klemm G. Peritoneal calcification and calciphylaxis. Nephron. 1989;51:124.

9. Fletcher S, Gibson J, Brownjohn AM. Peritoneal calcification secondary to severe hyperparathyroidism. Nephrol Dial Transplant. 1995;10:277-9.

10. Dejima K, Mitsuhashi H, Yasuda G, Hirawa N, Ikeda Y, Umemura S. Localization and extent of peritoneal calcification in three uremic patients on continuous ambulatory peritoneal dialysis. TherApher Dial. 2008;12(5):413-6.

11. Lucey BC, Varghese JC, Soto JA. Spontaneous hemoperitoneum: causes and significance. CurrProblDiagnRadiol. 2005;34(5):182-95.

12. Ikee R, Branch J, Honda K, Ishioka K, Oka M, Maesato K, et al. Recurrence of severe hemoperitoneum in a patient on peritoneal dialysis. Perit Dial Int. 2009;29(5):583-5.

13. Winchester DP, Seed RW, Bergan JJ, Conn J Jr. Jaundice, hemobilia and hemoperitoneum: consequences of hepatic artery aneurysm rupture. ProcInst Med Chic. 1969;27(10):280-1.

14. Nishiyama S, Zhu BL, Quan L, Tsuda K, Kamikodai Y, Maeda H. Unexpected suddendeath due to a spontaneous rupture of a gastric dissecting aneurysm: an autopsy case suggesting the importance of the double-rupture phenomenon. J ClinForensicMed. 2004;11(5):268-70.

15. Kawaguchi Y, Saito A, Kawanishi H, Nakayama M, Miyazaki M, Nakamoto H, et al. Recommendations on the management of encapsulating peritoneal sclerosis in Japan, 2005: diagnosis, predictive markers, treatment, and preventive measures. Perit Dial Int. 2005;25Suppl 4:S83-95.

16. Korte MR, Sampimon DE, Betjes MG, Krediet RT. Encapsulating peritoneal sclerosis: the state of affairs. Nat Rev Nephrol. 2011;7(9):528-38. 